\title{
AUMENTO DEL TRANSPORTE DE CALOR MEDIANTE ONDAS ELECTROMAGNÉTICAS SUPERFICIALES
}

\author{
INCREASE OF HEAT TRANSPORT BY SURFACE ELECTROMAGNETIC \\ WAVES
}

\author{
${ }^{1}$ Jose Ordonez Miranda \\ ${ }^{2}$ Laurent Tranchant \\ ${ }^{3}$ Satoki Hamamura \\ ${ }^{4}$ Tomohide Yabuki \\ ${ }^{5}$ Alejandro Vega
}

\author{
${ }^{6}$ Fernando Cervantes-Alvarez \\ ${ }^{7}$ Juan Jose Alvarado-Gil \\ ${ }^{8}$ Sebastian Volz \\ ${ }^{9}$ Koji Miyazaki
}

\section{RESUMEN}

Como es conocido, la conductividad térmica de una película delgada generalmente disminuye a medida que su espesor se reduce a través de valores nanométricos (Liu \& M. Asheghi, 2006); esto genera el sobrecalentamiento y la reducción de la vida útil de procesadores y otros componentes electrónicos (Pop, 2010). Sin embargo, dado que las películas más delgadas tienen mayores cocientes área/volumen, los predominantes efectos superficiales en nanopelículas permiten el transporte de energía térmica no solo dentro de sus volúmenes, sino también a lo largo de sus interfaces. En nanopelículas polares, este transporte superficial es impulsado por fonones-polaritones de superficie, los cuales son ondas electromagnéticas generadas por el acoplamiento de fonones y fotones a lo largo de sus superficies. Modelos teóricos predicen que estos polaritones pueden contribuir significativamente a la conductividad térmica en el plano de películas de $\mathrm{SiO}_{2}$ con espesores menores a $200 \mathrm{~nm}$ (Chen et al., 2005; OrdonezMiranda et al., 2013). En el presente trabajo demostramos experimentalmente este aumento de la conductividad térmica, mediante las técnicas $3 \omega$ y rejilla transitoria. Los resultados medidos a través de estas dos técnicas son consistentes y muestran que la conductividad térmica en el plano de una película de $\mathrm{SiO}_{2}$ de $20 \mathrm{~nm}$ de espesor a temperatura ambiente es el doble de su contraparte debida a fonones solamente. Mediciones adicionales de la difusividad térmica de películas de $\mathrm{SiO}_{2}$ revelan que esta propiedad térmica también aumenta para películas más delgadas, de tal manera que la relación (conductividad térmica)/(difusividad térmica) $=$ capacidad calorífica volumétrica se mantiene independiente del espesor de la película. Los resultados experimentales obtenidos aquí abren una nueva vía para desarrollar nanomateriales térmicamente conductores útiles para una refrigeración electrónica eficiente.

Palabras clave: Conductividad térmica en el plano, ondas electromagnéticas superficiales, refrigeración electrónica.

\footnotetext{
${ }^{1}$ Institut Pprime, CNRS, Université de Poitiers, ISAE-ENSMA, F-86962, Futuroscope, Chasseneuil, France. E-mail : jose.ordonez@ecp.fr ${ }^{2}$ Department of Mechanical and Control Engineering, Kyushu Institute of Technology, 1-1 Sensuicho, Tobata-ku, Kitakyushu 804-8550, Japan. ${ }^{3}$ Department of Mechanical and Control Engineering, Kyushu Institute of Technology, 1-1 Sensuicho, Tobata-ku, Kitakyushu 804-8550, Japan. ${ }^{4}$ Department of Mechanical and Control Engineering, Kyushu Institute of Technology, 1-1 Sensuicho, Tobata-ku, Kitakyushu 804-8550, Japan. ${ }_{5}^{5}$ Departamento de Física Aplicada, CINVESTAV-Unidad Mérida, Carretera Antigua a Progreso km 6, Cordemex, Mérida, Yucatán 97310, México. Email: avegaflick@gmail.com

${ }^{6}$ Departamento de Física Aplicada, CINVESTAV-Unidad Mérida, Carretera Antigua a Progreso km 6, Cordemex, Mérida, Yucatán 97310, México. Email:kafhes@hotmail.com

${ }^{7}$ Institut Pprime, CNRS, Université de Poitiers, ISAE-ENSMA, F-86962, Futuroscope, Chasseneuil, France. Departamento de Física Aplicada, CINVESTAV-Unidad Mérida, Carretera Antigua a Progreso km 6, Cordemex, Mérida, Yucatán 97310 , México. E-mail : juan.alvarado@cinvestav@mx

${ }^{8}$ LIMMS/CNRS-IIS (UMI2820), Institute of Industrial Science, University of Tokyo, 4-6-1 Komaba, Meguro-ku, Tokyo, 153-8505, Japan. Laboratoire d'Energétique Moléculaire et Macroscopique, Combustion, UPR CNRS 288, CentraleSupelec, Université Paris-Saclay, Bat. Eiffel, 3, rue Joliot Curie, 91192 Gif-sur-Yvette cedex-France.

${ }^{9}$ Department of Mechanical and Control Engineering, Kyushu Institute of Technology, 1-1 Sensuicho, Tobata-ku, Kitakyushu 804-8550, Japan.
} 


\section{ABSTRACT}

As is well known, the thermal conductivity of a thin film generally decreases when its thickness is reduced through nanometric values (Liu \& M. Asheghi, 2006), which generates overheating and the reduces the lifetime of processors and other electronic components (Pop, 2010). However, since thinner films have higher area/volume ratios, the predominant surface effects in nano-films allow the transport of thermal energy not only within their volumes, but also along their interfaces. In polar nanofilms, this surface transport is driven by surface phonon-polaritons, which are electromagnetic waves generated by the coupling of phonons and photons along their surfaces. Theoretical models predict that these polaritons can significantly contribute to the thermal conductivity in the plane of $\mathrm{SiO} 2 \mathrm{films}$ with thicknesses less than $200 \mathrm{~nm}$ (Chen et al., 2005, Ordonez-Miranda et al., 2013). In this work, we experimentally demonstrate this increase in thermal conductivity using the $3 \omega$ techniques and transient grid. The results measured by these two techniques are consistent and show that the in - plane thermal conductivity of a SiO 2 film of 20 nm thickness at room temperature is twice its counterpart due to phonons only. Additional measurements of the thermal diffusivity of $\mathrm{SiO} 2$ films reveal that this thermal property also increases for thinner films, in such a way that the relation (thermal conductivity) / (thermal diffusivity) = volumetric heat capacity is kept independent of the film thickness. The experimental results obtained here open a new way to develop thermally conductive nanomaterials useful for efficient electronic cooling.

Keywords: Electronic cooling electrónica, Surface electromagnetic waves, Thermal conductivity in the plane.

\section{INTRODUCCIÓN}

Los fonones-polaritones de superficie (FPS) pueden propagarse a lo largo de la superficie de nanomateriales dieléctricos polares, en distancias de alrededor de $1 \mathrm{~mm}$ (ver la Fig. 1), la cual es más de cuatro órdenes de magnitud más larga que las trayectorias libres medias típicas de fonones y electrones (Haynes, 2006). Dado que la conductividad térmica de un material depende directamente de esta distancia de propagación de sus portadores de energía, los FPS tienen el potencial para ser poderosos portadores de energía térmica en nanomateriales, tal como se predijo teóricamente en diferentes estructuras (Ordonez-Miranda et al., 2013; Gluchko et al., 2015). Experimentalmente, los FPS pueden ser excitados óptica o térmicamente. Para la excitación óptica, la superficie del material se ilumina con un rayo láser de una frecuencia dada $f$, lo cual genera FPS que se propagan con la misma frecuencia $f$, tal como establece los principios de conservación de la energía y del momento. Por el contrario, para la excitación térmica, el material se calienta y FPS en un amplio rango de frecuencias se excitan simultáneamente. Debido a la posibilidad de excitar un solo modo a la vez, el método óptico es más fácil para determinar la relación de dispersión y la longitud de propagación de estas ondas electromagnéticas de superficie (Agranovich \& Mills, 1982; Huber et al., 2005); en tanto que la observación de FPS térmicamente excitados requiere diferentes técnicas, tales como la rejilla de superficie (F. Marquier et al., 2004; Gluchko et al., 2017), la reflexión total atenuada (Agranovich \& Mills, 1982) o el microscopio de tunelización térmica (De Wilde et al., 2006).

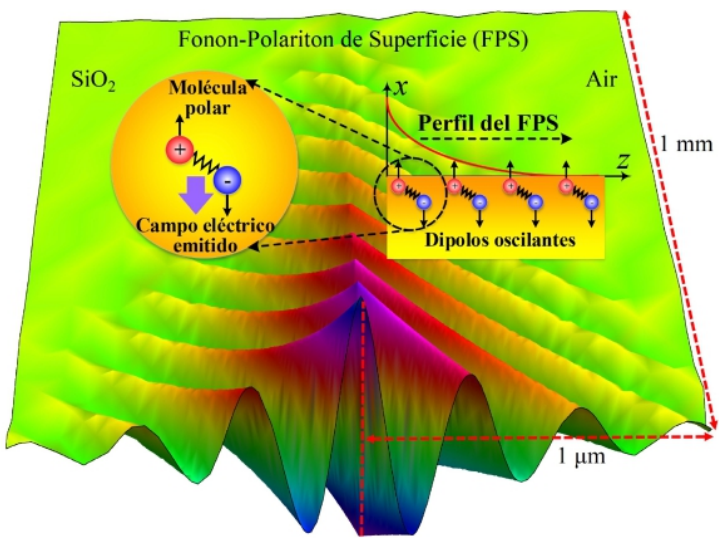

Figura 1. Generación y propagación de FPS a lo largo de una interface entre $\mathrm{SiO}_{2}$ y aire

La energía térmica transportada por FPS se ha medido para una red bidimensional de nanopartículas de $\mathrm{SiO}_{2}$ (Tervo et al., 2016) y para películas de grafeno encapsulado con nitruro de boro hexagonal (Tielrooij et al., 2018). En el primero de estos trabajos, el aumento de la conductividad térmica medida es parcialmente consistente con las predicciones del modelo de propagación balística de FPS, pero sus valores $(k=$ $0,010,03 \mathrm{~W} /(\mathrm{m} \cdot \mathrm{K})$ ) son insignificantes comparados con la contribución fonónica $(k=1,4 \mathrm{~W} /(\mathrm{m} \cdot \mathrm{K}))$. En el segundo trabajo, el flujo de calor en grafeno es aumentado debido al acoplamiento de sus portadores de energía con polaritones que se propagan en las interfaces del nitruro de boro; por lo tanto, este aumento no es debido a polaritones solamente. Por otro lado, ya ha sido predicho teóricamente el aumento de la conductividad térmica en el plano de películas delgadas 
de $\mathrm{SiO}_{2}$ que soportan la propagación de FPS, (Chen et al., 2005; Ordonez-Miranda et al., 2013; Yang et al., 1991); no obstante, aún no ha sido medida experimentalmente, lo que constituye el principal objetivo del presente trabajo. Tomando en cuenta que las propiedades térmicas de materiales dependen fuertemente de sus purezas, hemos decidido fabricar nuestras nanopelículas de $\mathrm{SiO}_{2}$ amorfo mediante el método de oxidación térmica (Yamane et al., 2002), el cual permite obtener películas ultra delgadas con alta pureza.

\section{MATERIALES Y MÉTODOS}

\section{Modelo teórico}

Consideremos una película delgada de espesor $d$, ancho $2 a$ y longitud $b$; tal como se muestra en la Fig. 2. La película está suspendida en aire, tiene una permitividad $\varepsilon(\omega)$ dependiente de la frecuencia $\omega$ de excitación y su espesor $d$ es considerado suficientemente pequeño $(d<300 \mathrm{~nm})$ como para ignorar su gradiente de temperatura en la dirección $z$. Para este caso - tomando como referencia a Chen et al. (2005) y a Ordonez-Miranda et al. (2013) — la ecuación de transporte de Boltzmann bajo la aproximación del tiempo de relajación predice que la contribución de FPS a la conductividad térmica en el plano de la película está dada por:

$$
\kappa=\frac{1}{4 \pi d} \int_{\omega_{\min }}^{\omega_{\max }} \hbar \omega \Lambda \beta_{R} \frac{\partial f_{0}}{\partial T} d \omega
$$

Donde $f_{0}$ es la distribución de Bose-Einstein; $T_{\text {es la }}$ temperatura promedio de la nanopelícula; $\operatorname{Re}(\beta)$ es la parte real del vector de onda $\beta$ de los FPS, cuya longitud de propagación es $\Lambda=[2 \operatorname{Im}(\beta)]^{-1}, 2 \pi \hbar$ es la constante de Planck, y $\omega_{\min }$ y $\omega_{\max }$ son las frecuencias mínimas y máximas que permiten la propagación de FPS a lo largo de las interfaces de la nanopelícula. Para el caso ideal de materiales no absorbentes de energía $(\operatorname{Im}(\varepsilon(\omega))=0)$ estas frecuencias están principalmente determinadas por la condición $\operatorname{Re}(\varepsilon(\omega))<-1$. Por otra parte, para un material absorbente $(\operatorname{Im}(\varepsilon(\omega)) \neq 0)$ como el $\mathrm{SiO}_{2}$, los FPS se propagan en un rango de frecuencia mucho más amplio que el de un material sin pérdidas, tal como se demostró recientemente de forma experimental (Gluchko et al., 2017). Tomando en cuenta la densidad de estados excitados de FPS propagándose en $\mathrm{SiO}_{2}$ a temperatura ambiente, el rango de integración de frecuencias en la Ec. (1) está dado por $\omega_{\min }=7,6$

Trad $/ \mathrm{s}$ y $\omega_{\max }=258,0 \mathrm{Trad} / \mathrm{s}$, para el $\mathrm{SiO}_{2}$ considerado en este trabajo. Una vez fijado este rango de integración, la Ec. (1) establece que $\kappa$ se encuentra determinada por la relación de dispersión $\beta=\beta(\omega)$, la cual se da por la siguiente solución de las ecuaciones de Maxwell del electromagnetismo para la polarización magnética transversal requerida en la existencia de
FPS (Ordonez-Miranda et al., 2013):

$$
p_{0}+\frac{p}{\varepsilon} \tanh \left(\frac{p d}{2}\right)=0
$$

Donde $p_{0}=\sqrt{\beta^{2}-(\omega / c)^{2}} \quad$ y $\quad p=$ $\sqrt{\beta^{2}-\varepsilon(\omega)(\omega / c)^{2}}$ siendo $c$ la velocidad de la luz en el vacío. La ecuación trascendente (2) será resuelta numéricamente para $\beta(\omega)$ mediante el software Wolfram Mathematica, usando como datos de entrada la permitividad compleja $\varepsilon(\omega)$ de $\mathrm{SiO}_{2}$ reportada en la literatura (Ordonez-Miranda et al., 2013; Palik, 1985).

Considerando que la longitud de propagación intrínseca $\Lambda$ de FPS puede ser del orden de $1 \mathrm{~mm}$ y que su propagación se encuentra limitada por el semiancho $a$ de nuestras muestras, como se revela en la Fig. 2, la longitud de propagación efectiva $\Lambda_{e}$ usada en la Ec. (1) a fin de determinar la conductividad térmica de PFS ha sido calculada con la regla de Mathiessen (Ordonez-Miranda et al., 2013) $\Lambda_{e}^{-1}=\Lambda^{-1}+a^{-1}$.Esta relación es válida cuando las ondas superficiales solo se propagan en esa dirección y si la temperatura del hilo calentador se mantiene relativamente uniforme a lo largo de su longitud, lo que se espera resulte cierto. Sin embargo, si el gradiente de temperatura a lo largo de este hilo es significativo, la excitación de estas ondas no es uniforme; en consecuencia, ellas también pueden propagarse en una dirección diagonal dentro del plano $\mathrm{x}-\mathrm{y}$. En este último caso, la longitud de propagación efectiva $\Lambda_{e}^{\prime}$ es la distancia promedio con que los FPS pueden propagarse en todas las direcciones posibles (Fig. 2), la cual se establece por:

$$
\frac{1}{\Lambda_{e}^{\prime}}=\frac{1}{\Lambda}+\frac{1}{2 a} \frac{\ln \left[1+(b / 2 a)^{2}\right]}{\sqrt{1+(b / 2 a)^{2}}-1}
$$

Las dos longitudes de propagación $\Lambda_{e}$ (Modelo I) y $\Lambda_{e}^{\prime} \quad$ (Modelo II) nos permitirán calcular dos valores para la conductividad térmica de FPS -Ec. (1)-, cuya comparación con sus correspondientes valores experimentales nos mostrará si la energía de estas ondas electromagnéticas se ve determinada por el semi-ancho $a$ de nuestras películas o también por su longitud $b$, tal como lo establece la Ec. (3).

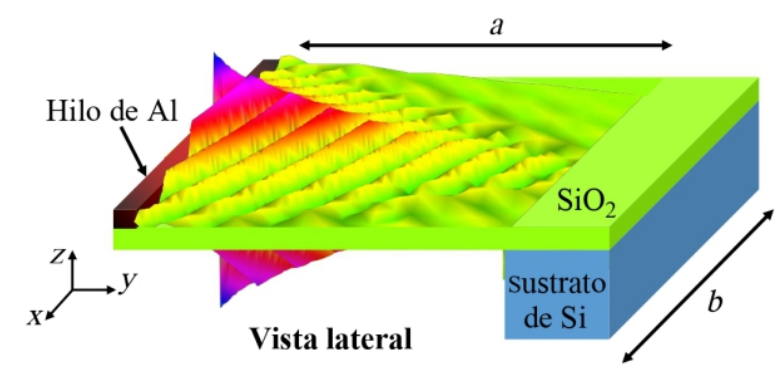




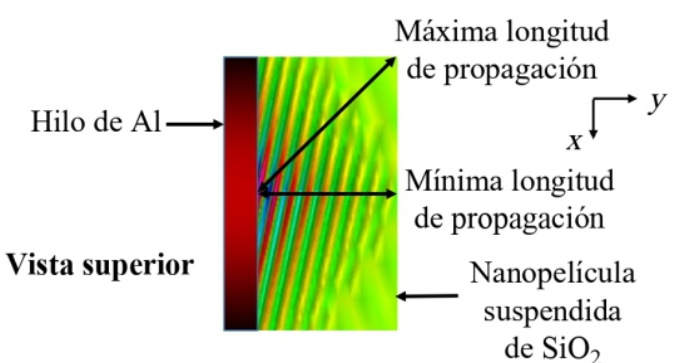

Figura 2. Esquema de la mitad de una película de $\mathrm{SiO}_{2}$ suspendida en aire. Las ondas superficiales se excitan térmicamente con el hilo de aluminio (Al) caliente colocado en el centro de la película y se propagan a lo largo de ambos lados, antes de dispersarse en sus bordes. La vista superior muestra la longitud de propagación mínima (modelo I) y máxima (modelo II), que se usaron en los cálculos teóricos.

\section{Fabricación de muestras}

Varias nanopelículas de $\mathrm{SiO}_{2}$ suspendidas en aire han sido fabricadas a través del método de oxidación térmica, que consiste en 12 pasos mostrados en la Fig. 3. En el paso I, el $\mathrm{SiO}_{2}$ es crecido sobre obleas de silicio pulido tipo $\mathrm{p}$ (100) de 2 pulgadas de diámetro y $300 \mu \mathrm{m}$ de espesor, en un horno a $1078{ }^{\circ} \mathrm{C}$ y utilizando $\mathrm{NH}_{3}$ como catalizador para espesores mayores de $50 \mathrm{~nm}$. Luego, se rocía una capa de aluminio de $100 \mathrm{~nm}$ de espesor sobre las muestras (paso II) y se deposita el fotorresistor utilizado para el gravado del alambre de aluminio (paso III). Las muestras se exponen luego a los rayos UV antes del revelado (paso IV) y la capa de aluminio no protegida es grabada mediante ácido fosfórico (paso V). Para reducir el estrés compresivo de las nanopelículas de $\mathrm{SiO}_{2}$, depositamos una capa de nitruro de silicio de $500 \mathrm{~nm}$ de espesor sobre y bajo ellas (paso VI), mediante la técnica de deposición de vapores químicos mejorados por plasma (PECVD). Se incorpora nuevamente la capa fotorresistente, se expone a los rayos UV y se graba la capa de nitruro de silicio posterior por grabado iónico reactivo (RIE) (pasos VII-IX).

Después de la eliminación de la fotoprotección, se añade tres capas de dióxido de silicio y una capa de nitruro de silicio para proteger la parte superior durante el grabado en húmedo del sustrato (paso X). Las dos capas de $\mathrm{SiO}_{2}$ de $500 \mathrm{~nm}$ de espesor se depositan por PECVD y son muy porosas; sin embargo, la capa de vidrio de agua de $250 \mathrm{~nm}$ de espesor llena la porosidad de la capa inferior y refuerza la protección de la parte superior. El grabado en húmedo del sustrato se realizó con una solución acuosa de TMAH (hidróxido de tetrametilamonio) (paso XI), antes del grabado en seco por RIE de las capas protectoras superiores (paso XII). Con la finalidad de evitar el grabado excesivo, se mide los espesores restantes de las capas protectoras superiores para estimar correctamente el tiempo de grabado.

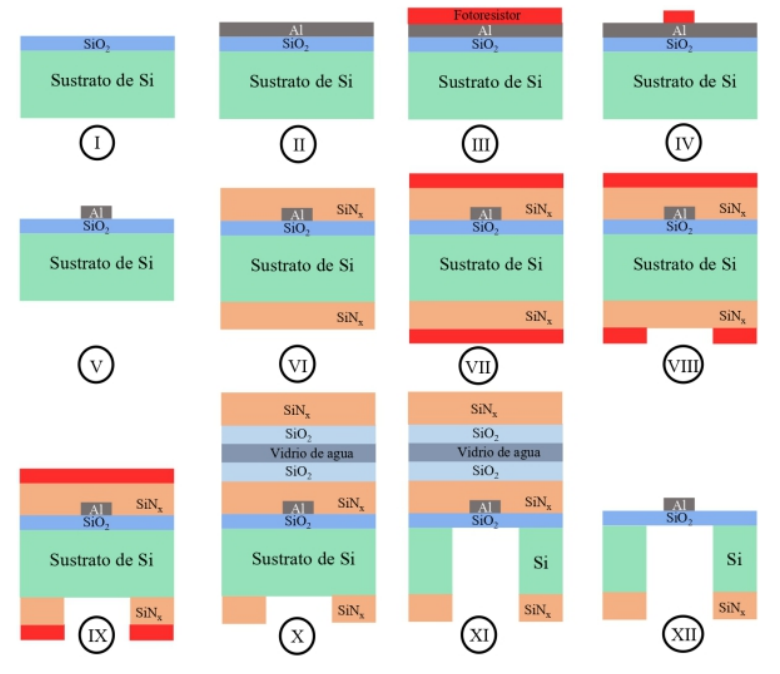

Figura 3. Proceso de fabricación por oxidación térmica de nanopelículas de $\mathrm{SiO}_{2}$ suspendidas en aire

El espesor entre $300 \mathrm{~nm}$ y $20 \mathrm{~nm}$ de las nanopelículas suspendidas se midieron mediante reflectancia espectroscópica en los bordes de las muestras. Este rango de espesores es lo suficientemente amplio como para resaltar y cuantificar de manera completa la energía térmica de FPS, sin ambigüedades, tal cual lo predicen estudios teóricos previos (Chen et al., 2005; Ordonez-Miranda et al., 2013). Para las medidas de $3 \omega$, las dimensiones de los hilos de aluminio utilizados como calentadores $(100 \mathrm{~nm}$ de espesor y $6 \mu \mathrm{m}$ de ancho) y de las nanopelículas rectangulares de $\mathrm{SiO}_{2}$ (250700 $\mu \mathrm{m}$ de ancho y $45 \mathrm{~mm}$ de largo) fueron elegidas cuidadosamente a fin de minimizar las pérdidas de calor - como se muestra en la Fig. 4-. Por otro lado, para las mediciones de la rejilla transitoria, se depositó una capa delgada de $\operatorname{AuPd}(2,8 \mathrm{~nm})$ en cada muestra de $\mathrm{SiO}_{2}$ y así garantizar la conversión de la luz láser en calor en la superficie iluminada de la muestra. (a)

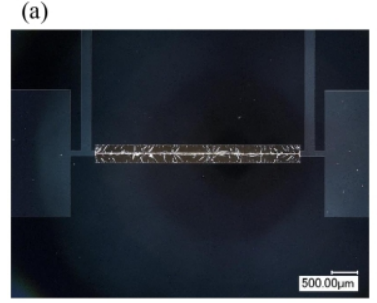

(b)

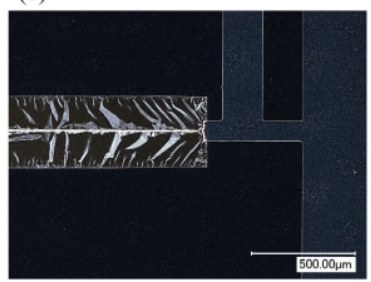

(c)

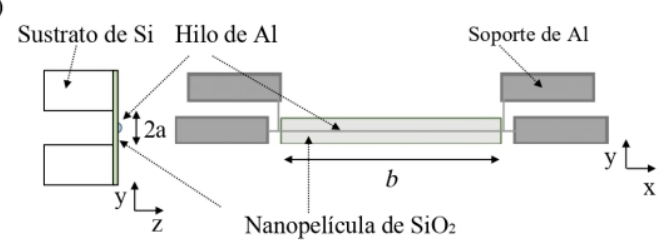

Vista Lateral

Figura 4. Nanopelícula de $\mathrm{SiO}_{2}$ fabricada con un hilo Al. Las imágenes (a) y (b) revelan una vista superior de una película suspendida de $30 \mathrm{~nm}$ de espesor, para dos aumentos diferentes; en cambio (c) exhibe las vistas superior y lateral de un esquema de las muestras. Durante las mediciones de 3, utilizamos cuatro soportes de Al para cuantificar por separado 
la intensidad de la corriente (dos inferiores) y el voltaje (dos superiores) de la corriente eléctrica que fluye a lo largo del hilo de Al.

\section{Medición de la conductividad térmica}

\section{A. Método $3 \omega$}

El primer método utilizado para determinar la conductividad térmica y la difusividad térmica en el plano de las nanopelículas de $\mathrm{SiO}_{2}$ está basado en el método $3 \omega$ reportado por Jain \& Goodson (2008). Dicho método consiste en calentar cada nanopelícula mediante un hilo de Al depositado en su centro (Fig. $4[\mathrm{~b}])$ por donde se hace circular una corriente eléctrica. Este hilo también se usa como sensor de temperatura debido a que su resistencia eléctrica depende linealmente de la temperatura, como se muestra en la Fig. 5(a). Cuando se aplica un voltaje continuo (DC) al hilo, su aumento promedio de temperatura está dado por:

$$
\bar{\theta}_{D C}=\frac{4 R I_{0}^{2}}{\kappa d} \sum_{n=0}^{\infty} \frac{\tanh [(2 n+1) \pi a / b]}{[(2 n+1) \pi]^{3}},
$$

Donde $R$ es la resistencia eléctrica del hilo a temperatura ambiente e $I_{0}$ es la intensidad de la corriente eléctrica. La ecuación (4) indica que la conductividad térmica $\kappa$ en el plano se puede extraer fácilmente de la gráfica entre la potencia eléctrica de entrada $R I_{0}^{2}$ y el aumento de temperatura DC (Fig. $5[\mathrm{~b}])$, que se obtiene a partir del voltaje medido a través del cable metálico.
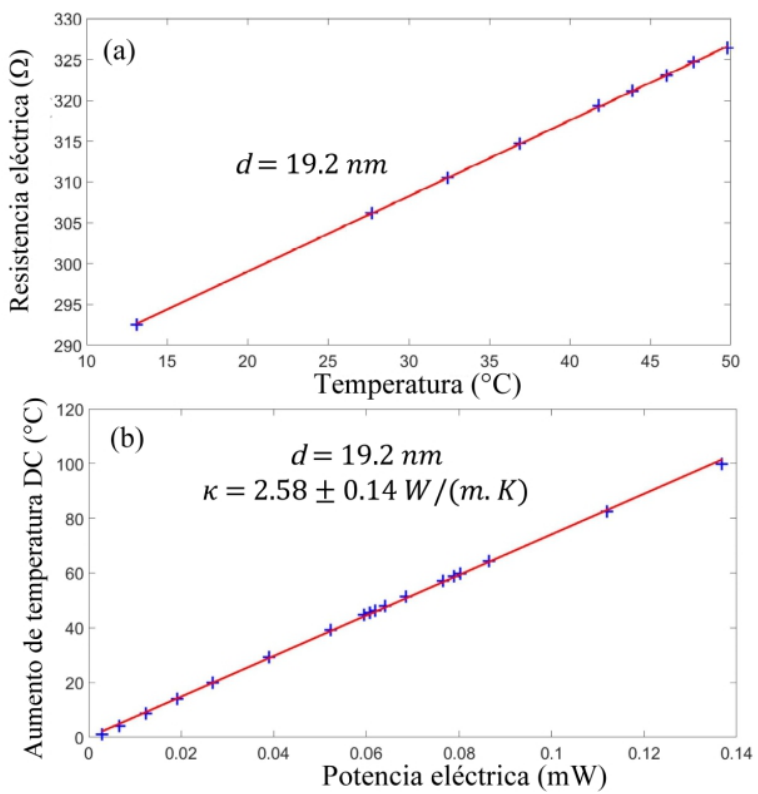

Figura 5. (a) Variación de la resistencia eléctrica del hilo de aluminio depositado en una película de $\mathrm{SiO}_{2}$ de 19,2 nm de espesor, en función de su temperatura (b) Aumento de la temperatura del hilo en función de la potencia eléctrica. El buen ajuste de la Ec. (4) a los datos experimentales nos permite determinar la conductividad térmica de la muestra.

Al aplicar un voltaje oscilatorio (AC) de frecuencia al hilo, el aumento de su temperatura tiene un componente DC asociado a la ecuación (4) y otro componente AC, cuya amplitud promedio en un período está dada — según Jain \& Goodson (2008) por la siguiente ecuación:

$$
\left|\bar{\theta}_{A C}\right|=\frac{2 R I_{0}^{2}}{\kappa d b}\left|\sum_{n=0}^{\infty} \frac{\tanh \left[\Delta_{2 n+1} a\right]}{\Delta_{2 n+1}[(2 n+1) \pi]^{2}}\right|,
$$

Donde

$$
\Delta_{2 n+1}=\sqrt{\left(\frac{(2 n+1) \pi}{b}\right)^{2}+\left(\frac{2 i \omega}{\alpha}\right)^{2}} .
$$

$\left|\bar{\theta}_{A C}\right|$ es la amplitud del componente $2 \omega$ de la temperatura y es directamente proporcional al componente $3 \omega$ del voltaje a través del hilo. Una vez que la conductividad térmica haya sido determinada con la Ec. (4), la ecuación (5) se utiliza para establecer la difusividad térmica $\alpha$ de las muestras, a partir de la figura $\left|\bar{\theta}_{D C}\right|$ versus $R I_{0}$ determinada experimentalmente (Fig. 6).

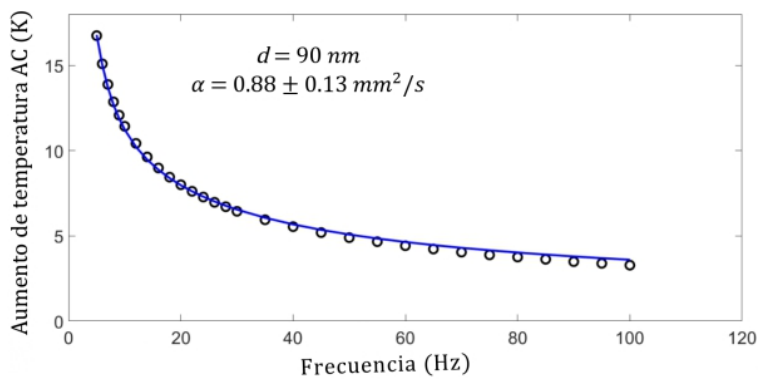

Figura 6. Componente $\mathrm{AC}$ de temperatura en función de la frecuencia de la corriente que circula por el hilo de calentamiento de una película de $90 \mathrm{~nm}$ de espesor. El buen ajuste de la Ec. (5) a los datos experimentales (puntos negros) nos permite determinar la difusividad térmica de la muestra.

\section{A. Método de la rejilla transitoria}

Con el fin de corroborar los resultados obtenidos con el método $3 \omega$, realizamos mediciones utilizando la técnica de rejilla transitoria inducida por láser (Eichler, 1986). En esta técnica de resolución temporal dos pulsos cortos de laser ( $8 \mathrm{~ns}$ ) se cruzan en un ángulo $2 \theta$ para generar un patrón de intensidad con un periodo $\Lambda=\lambda \sin (2 \theta)$, donde $\lambda$ es la longitud de onda óptica —Figuras 7(a) y 7(b) - . La absorción de la luz del láser por la muestra crea un perfil de temperatura espacialmente periódica, que se mantiene hasta cuando la energía térmica se redistribuya y alcance el equilibrio térmico - Figura 7(b)—. Esta disminución de la temperatura induce cambios en el índice de refracción, los cuales son monitoreados a través de la difracción de un láser de onda continua. En el caso de nuestras nanopelículas de $\mathrm{SiO}_{2}$ suspendidas en aire, el perfil de temperatura inducido decae como una función exponencial (Eichler, 1986; Vega-Flick et al., 2016):

$$
T(t)=T_{0} e^{-4 \pi^{2} \alpha t / \Lambda^{2}},
$$

Donde es el tiempo y $\alpha$ es la difusividad térmica de la nanopetícula. La determinación experimental de la 
función $T(t)$ ha sido utilizada para establecer $\alpha$ a través de un proceso de ajuste.
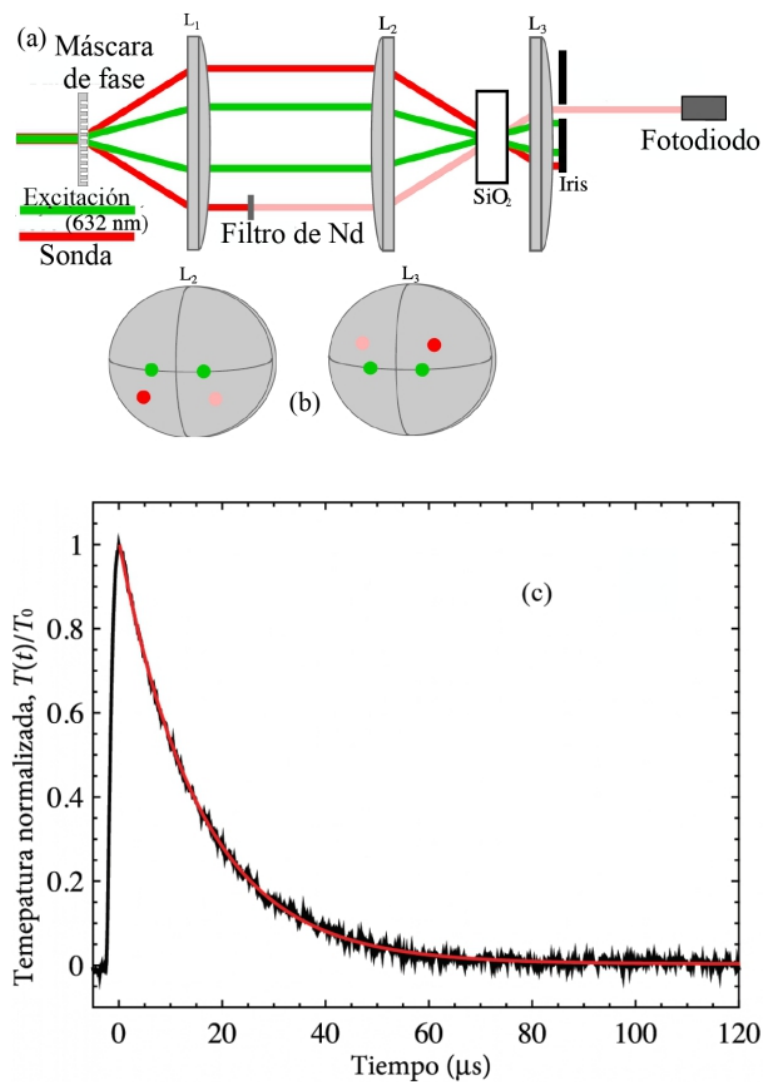

Figura 7. (a) Montaje experimental del método de la rejilla transitoria con detección óptica para muestras de $\mathrm{SiO}_{2}$. Las lentes $\mathrm{L}_{1}, \mathrm{~L}_{2}$ y $\mathrm{L}_{3}$ tienen una distancia focal de $5 ; 15$ y $7,5 \mathrm{~cm}$, respectivamente. (b) Vista frontal de las lentes $\mathrm{L}_{2} \mathrm{y} \mathrm{L}_{3}$, donde el par de haces de la sonda tiene un desplazamiento vertical con respecto a la excitación para permitir la medida de la temperatura. (c) Señal típica obtenida para una película de $\mathrm{SiO}_{2}$ de $140 \mathrm{~nm}$ de espesor utilizando un período de rejilla de $\Lambda=32 \mu \mathrm{m}$. La línea roja continua representa el mejor ajuste de laEc. (7).

\section{RESULTADOS Y DISCUSIONES}

La conductividad térmica $(\kappa)$ y la difusividad térmica $(\alpha)$ de nuestras películas de $\mathrm{SiO}_{2}$ medidas a través del método $3 \omega$ aparecen en las Figs. 8(a) y 9(a), respectivamente, como funciones de sus espesores. Para espesores de alrededor de $d \approx 300 \mathrm{~nm}$, tanto $\kappa_{\text {como }}$ $\alpha$ tienen valores similares a sus correspondientes contrapartes de materiales macroscópicos, lo que indica la ausencia de polaritones para esos espesores relativamente grandes. Sin embargo, para $d<50 \mathrm{~nm}$, ambas propiedades exhiben un aumento significativo de más del 50\%, el cual representa la huella digital de polaritones debido a las siguientes razones: i) $\quad \kappa$ y $\alpha$ aumentan para nanopelículas más delgadas, tal como lo establece la creciente influencia de los efectos de superficie, a medida que los espesores de las películas disminuye. ii) La conductividad térmica de $\mathrm{SiO}_{2}$ macroscópico solo aumenta alrededor de 0,17
$\mathrm{W} /(\mathrm{m} \cdot \mathrm{K})(12 \%)$ (Zeller \& Pohl, 1971) cuando su temperatura aumenta de $289 \mathrm{~K}$ a $381 \mathrm{~K}$, comparable o superior al aumento de temperatura experimentado por el hilo calentador de Al utilizado en nuestros experimentos $3 \omega$.
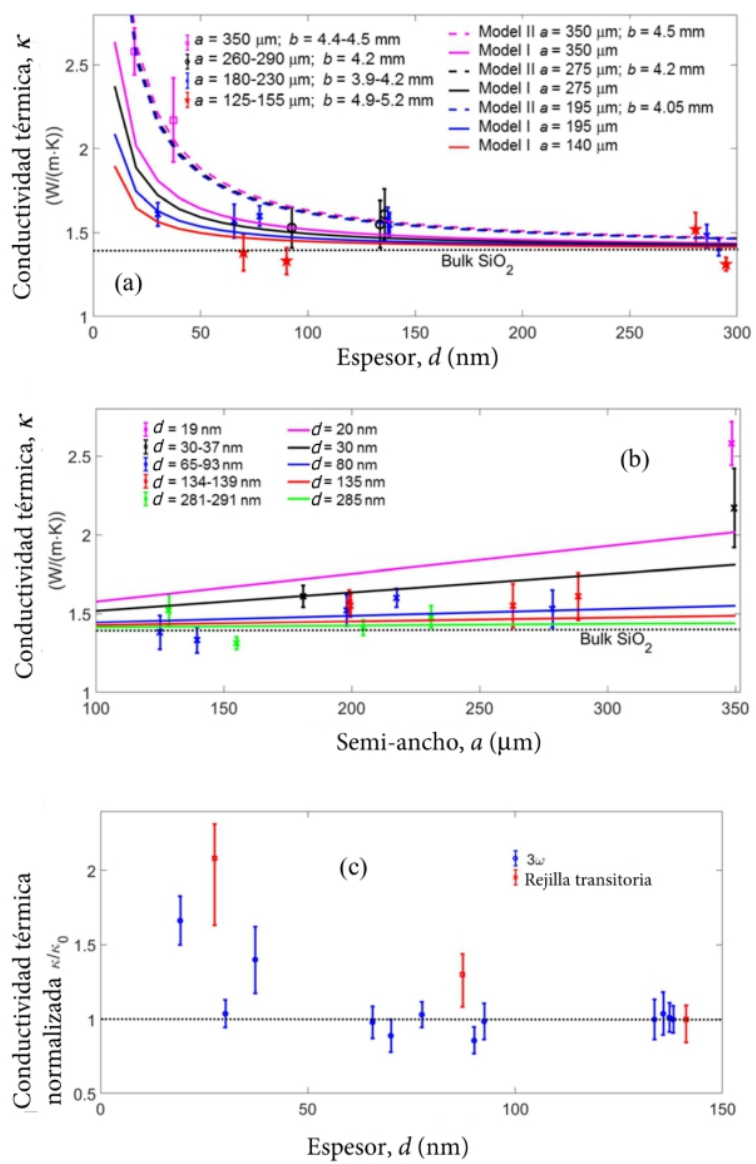

Figura 8. Conductividad térmica de nanopelículas de $\mathrm{SiO}_{2}$ en función de sus (a) espesores y (b) semi-anchos. Los datos experimentales obtenidos con el método $3 \omega$ están comparados con las predicciones teóricas de la Ec. (1), considerando que la propagación de las ondas superficiales está limitada por el semi-ancho de la película (modelo I) o bien, ocurre en todas las direcciones del plano de la película (modelo II). La línea punteada representa la conductividad térmica del $\mathrm{SiO}_{2}$ macroscópico a temperatura ambiente (Haynes, 2006; Yamane et al., 2002). (c) Conductividad térmica normalizada medida por los métodos $3 \omega$ y reja transitoria, en función del espesor de la nanopelícula. Los valores $\kappa_{0}=1,55 \mathrm{~W} /(\mathrm{m} \cdot \mathrm{K})(d=138,1 \mathrm{~nm})$ y $\kappa_{0}=3,37 \mathrm{~W} /(\mathrm{m} \cdot \mathrm{K})$ $(d=141,2 \mathrm{~nm})$ se han utilizado para la normalización de los datos obtenidos a través de los métodos 3 y rejilla transitoria, respectivamente.

Las predicciones teóricas que se muestran en la Fig. 8(a) fueron calculadas con la ecuación (1), donde se utilizaron las longitudes de propagación efectivas $\Lambda_{e}$ (modelo I) y $\Lambda_{e}^{\prime} \quad$ (modelo II). Considerando que nuestras mediciones experimentalmente representan la conductividad térmica total (fonones + polaritones), a las predicciones de la ecuación (1) se ha agregado la conductividad térmica del $\mathrm{SiO}_{2}[1,4 \mathrm{~W} /(\mathrm{m} \cdot \mathrm{K})]$ 
macroscópico, con la finalidad de comparar adecuadamente los valores experimentales y teóricos de la conductividad térmica. Nótese que las predicciones del modelo I coinciden con los valores experimentales de para nanopelículas con un ancho $a<230 \mu \mathrm{m}$; en tanto que las medidas para muestras más anchas se describen mejor con el modelo II. Esto es razonable ya que las nanopelículas más anchas tienen una mayor densidad de arrugas superficiales, lo que desvía el transporte de calor de la dirección $y$ asumida por el modelo I. La transición entre estos dos comportamientos se produce para $250 \mu \mathrm{m}<a<300$ $\mu \mathrm{m}$, en el que ambos modelos predicen prácticamente los mismos valores (líneas negras sólidas y discontinuas). La propagación de ondas electromagnéticas de superficie a lo largo de películas estrechas ( $a 230 \mu \mathrm{m})$ se ve limitada por su semi-ancho $a$, mientras que a lo largo de películas anchas $(a>300$ $\mu \mathrm{m})$, dicha propagación también está limitada por su longitud $b$. Las desviaciones relativamente pequeñas entre los valores experimentales y los valores teóricos se pueden asociar a la pureza de las películas de $\mathrm{SiO}_{2}$ y a sus arrugadas superficies; esto puede agregar algunas distorsiones de propagación que son difíciles de predecir. Teniendo en cuenta que la mayoría de nuestros datos experimentales están bien descritos por el modelo I, a partir de ahora, vamos a considerar que el semi-ancho a de las nanopelículas es la distancia de propagación principal de las ondas electromagnéticas de superficie a lo largo de nuestras muestras.
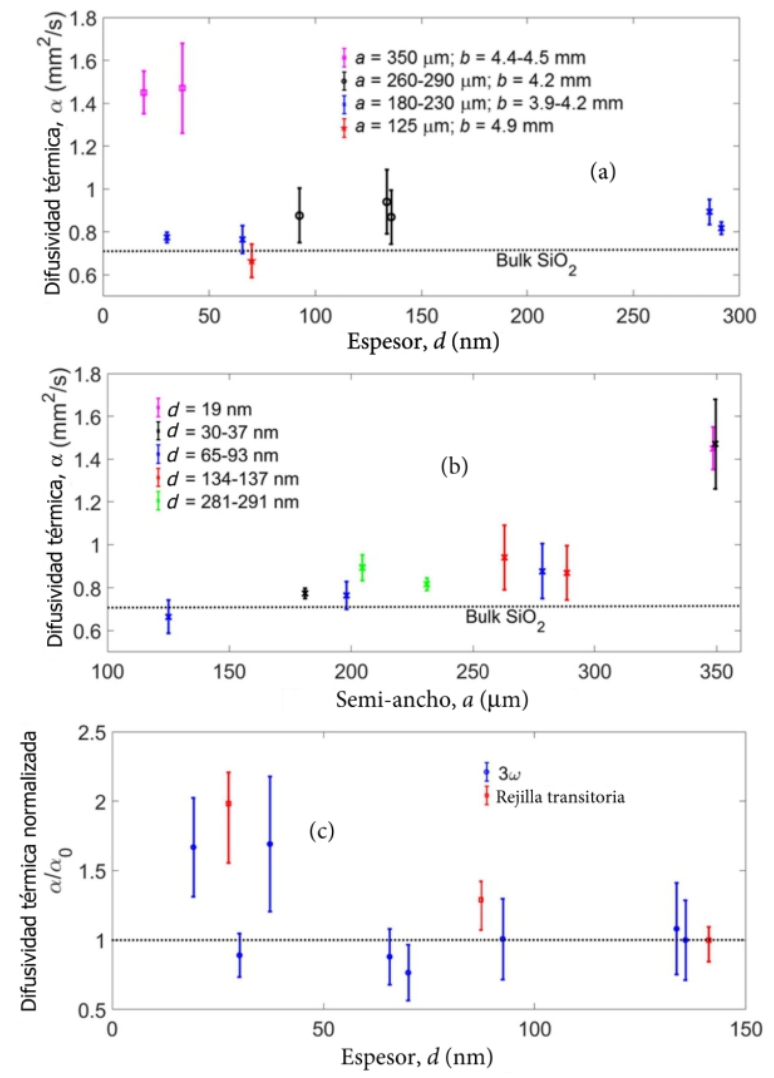

Figura 9. Difusividad térmica de nanopelículas de $\mathrm{SiO}_{2}$ en función de sus (a) espesores y (b) semi-anchos. La línea punteada representa la difusividad térmica del $\mathrm{SiO}_{2}$ macroscópico a temperatura ambiente (Haynes, 2006; Yamane et al., 2002). (c) Difusividad térmica normalizada medida por los métodos $3 \omega$ y rejilla transitoria, en función del espesor de la nanopelícula. Los valores $\alpha_{0}=0,87 \mathrm{~mm}^{2} / \mathrm{s}(d$ $=138,1 \mathrm{~nm})$ y $\alpha_{0}=1,69 \mathrm{~mm}^{2} / \mathrm{s}(d=141,2 \mathrm{~nm})$ se han utilizado para la normalización de los datos obtenidos por los métodos $3 \omega$ y rejilla transitoria, respectivamente.

El aumento de $\kappa$ en la Fig. 8(a) no es monótono a medida que el espesor de la nanopelícula se reduce, esto se debe a su dependencia del ancho $a$ mostrada en la Fig. 8(b). Para espesores menores a $40 \mathrm{~nm}(37 \mathrm{~nm}, 30$ $\mathrm{nm}$ y $19 \mathrm{~nm}$ ), podemos observar claramente dos efectos: cuando $a$ se mantiene constante, $\kappa$ aumenta para nanopelículas más delgadas, mientras que para espesores similares $(30-37 \mathrm{~nm}), \kappa$ disminuye dramáticamente a medida que la nanopelícula se estrecha. Esta tendencia es difícil de observar experimentalmente para espesores mayores de $50 \mathrm{~nm}$, en razón de la propagación limitada de las ondas superficiales, del mismo modo en que lo establecen las predicciones teóricas. Por otro lado, el aumento de la conductividad térmica también está presente en la difusividad térmica, como se muestra en las Figs. 9(a) y 9(b). La consistencia entre las mediciones de $\kappa$ y $\alpha$ se ve reformada por su cociente (capacidad calorífica volumétrica) $\quad \kappa / \alpha \approx 1,8 \mathrm{MJ} /\left(\mathrm{m}^{3} \cdot \mathrm{K}\right) \quad$ que es prácticamente independiente del espesor de la nanopelícula, tal como está reportado en la literatura para el $\mathrm{SiO}_{2}$ a temperatura ambiente (Haynes, 2006).

El aumento correlacionado de $\kappa$ y $\alpha$, medido por el método $3 \omega$, está confirmado por el método de rejilla transitoria, tal cual se observa en las Figs. 8(c) y 9(c), respectivamente. Los valores de $\kappa$ y $\alpha$ cuantificados para nanopelículas con espesores de 141,2 nm, 87,3 nm y $27,5 \mathrm{~nm}$ son más altos que sus correspondientes valores obtenidos por el método $3 \omega$ para nanopelículas de espesores similares, debido a la nanocapa de AuPd $(2,8 \mathrm{~nm})$ depositada sobre estas tres muestras y a las diferencias en el ancho y la longitud de las muestras. Sin embargo, al normalizar los valores de $\quad \kappa$ y $\alpha$ obtenidos por cada técnica con sus correspondientes valores $\left(\kappa_{0} \mathrm{y} \alpha_{0}\right)$ medidos para una película gruesa $(d 300 \mathrm{~nm})$, observamos que ambas técnicas producen un aumento consistente de $\kappa / \kappa_{0}$ y $\alpha / \alpha_{0}$, lo cual representa la huella digital de las ondas electromagnéticas de superficie. Curiosamente, los valores relativamente altos de $\kappa$ y $\alpha$ calculados con el método de la rejilla transitoria, sugieren que estas ondas electromagnéticas son capaces de acoplarse con los electrones libres de la nanocapa de AuPd altamente conductora para mejorar aún más su transporte de energía térmica. Este último resultado puede abrir una nueva vía para los estudios teóricos y experimentales de la propagación de ondas electromagnéticas de superficie a lo largo de las interfaces entre materiales polares y metálicos. 


\section{CONCLUSIONES}

En base a los métodos $3 \omega$ y rejilla transitoria, hemos medido experimentalmente la conductividad térmica y difusividad térmica de ondas electromagnéticas de superficie que se propagan a lo largo de nanopelículas de $\mathrm{SiO}_{2}$ suspendidas en aire. Se ha demostrado que ambas propiedades térmicas aumentan más del 50\% cuando sus espesores se reducen de $300 \mathrm{~nm}$ a $50 \mathrm{~nm}$. Este aumento significativo representa la huella digital de la energía de estas ondas superficiales, cuya distancia de propagación y energía aumentan con la anchura de una nanopelícula. Los resultados experimentales obtenidos por ambas técnicas son consistentes entre ellos y con las predicciones teóricas para la conductividad térmica de las ondas electromagnéticas de superficie y, por lo tanto, demuestran la existencia de un nuevo canal (superficial) para el transporte de calor a lo largo de nanomateriales polares.

\section{AGRADECIMIENTOS}

Este trabajo ha sido parcialmente financiado por la Cellule Énergie del CNRS a través el proyecto $\mathrm{N}^{\circ}$ 267745, por la Fundación Japonesa para la Ciencia y Tecnología mediante el proyecto $\mathrm{N}^{\circ} \mathrm{JP} 16 \mathrm{~K} 18031$ y por el Conacyt mexicano a través del proyecto Fronteras de la Ciencia $\mathrm{N}^{\circ} 192$.

\section{REFERENCIAS BIBLIOGRÁFICAS}

Agranovich, V. M. et Mills, D. L. (1982). Surface Polaritons: Electromagnetic Waves at Surface and Interfaces. Amsterdam, The Netherlands: Elsevier Science.

Chen, D.-Z., Narayanaswamy, A. et Chen, G. (2005). Surface phonon-polariton mediated thermal conductivity enhancement of amorphous thin films. Physical Review B, 72, 155435-155438.

De Wilde, Y., Formanek, F., Carminati, R., Gralak, B., Lemoine, P.-A., Joulain, K. Mulet, J.-P., Chen, Y. et Greffet, J.-J. (2006). Thermal radiation scanning tunnelling microscopy. Nature, 444, 740-743.

Eichler, H. J., Gunter, P. et Pohl, D. W. (1986). Laserinduced dynamic gratings. Berlin, Germany: Springer.

Gluchko, S., Ordonez-Miranda, J., Tranchant, L., Antoni, T. et Volz, S. (2015). Focusing of surface phonon-polaritons along conical and wedge polar nanostructures. Journal of Applied Physics, 118, 064301-064308.

Gluchko, S., Palpant, B., Volz, S., Braive, R. et Antoni, T. (2017). Thermal excitation of broadband and long-range surface waves on $\mathrm{SiO} 2$ submicron films. Applied Physics Letters,
110,263108-263111.

Haynes, W. M. 2006. CRC handbook of chemistry and physics. Florida, The United States: CRC press.

Huber, A., Ocelic, N., Kazantsev, D. et Hillenbrand, R. (2005). Near-field imaging of mid-infrared surface phonon polariton propagation. Applied physics letters, 87, 81103-81105.

Jain, A. et Goodson, K. E. (2008). Measurement of the thermal conductivity and heat capacity of freestanding shape memory thin films using the 3 method. Journal of Heat Transfer, 130, 102402-102408.

Liu, W. et Asheghi, M. (2006). Thermal conductivity measurements of ultra-thin single crystal silicon layers. Journal of heat transfer, 128, $75-83$.

Marquier, F., Joulain, K., Mulet, J.-P., Carminati, R., Greffet, J.-J. et Chen, Y. (2004). Coherent spontaneous emission of light by thermal sources. Physical Review B, 69, 155412-155422.

Ordonez-Miranda, J., Tranchant, L. Kim, B. Chalopin, Y., Antoni, T. et Volz, S. (2014). Effects of anisotropy and size of polar nano thin films on their thermal conductivity due to surface phonon-polaritons. Applied Physics Express, 7, 035201-035204.

Ordonez-Miranda, J., Tranchant, L., Tokunaga, T., Kim, B., Palpant, B., Chalopin, Y., Antoni, T. et Volz, S. (2013). Anomalous thermal conductivity by surface phonon-polaritons of polar nano thin films due to their asymmetric surrounding media. Journal of Applied Physics, 113, 084311-084318.

Palik, E. D. (1985). CRC Handbook of Optical Constants of Solids. Orlando, The United States: Academic Press.

Pop, E. (2010). Energy dissipation and transport in nanoscale devices. Nano Research, 3, $147-169$.

Tervo, E. J., Adewuyi, O. S., Hammonds, J. S. et Cola, B. A. (2016). High thermal conductivity in polaritonic $\mathrm{SiO}_{2}$ nanoparticle beds. Materials Horizons, 3, 434-441.

Tielrooij, K. J., Hesp, N. C. H., Principi, A., Lundeberg, M. B., Pogna, E. A. A., Banszerus, L. Mics, Z., Massicotte, M., Schmidt, P. Davydovskaya, D., Purdie, D. G., Goykhman, I., Soavi, G. Lombardo, A., Watanabe, K., Taniguchi, T. Bonn, M., Turchinovich, D., Stampfer, C., Ferrari, A. C., Cerullo, G. Polini, M. et Koppens, F. H. L. (2018). Out-of-plane heat transfer in van der Waals stacks through electronhyperbolic phonon coupling. Nature Nanotechnology, $13,41-46$.

Vega-Flick, A., Duncan, R. A., Eliason, J. K., Cuffe, J., Johnson, J. A., Peraud, J.-P. M., Zeng, L., Lu, 
Z., Maznev, A. A., Wang, E. N., Alvarado-Gil, J. J., Sledzinska, M., Sotomayor Torres, C. M., Chen, G. et Nelson, K. A. (2016). Thermal transport in suspended silicon membranes measured by laser-induced transient gratings. AIP Advances 6, 121903-121915.

Yamane, T., Nagai, N., Katayama, S.-I. et Todoki, M. (2002). Measurement of thermal conductivity of silicon dioxide thin films using a 3 method. Journal of Applied Physics, 91, 9772-9776.

Yang, F., Sambles, J. R. et Bradberry, G. W. (1991). Long-range surface modes supported by thin films. Physical Review B, 44, 5855-5872.

Zeller, R. C. et Pohl, R. O. (1971). Thermal conductivity and specific heat of noncrystalline solids. Physical Review B, 4, 2029-2041. 